\title{
Autism risk following antidepressant medication during pregnancy
}

\author{
A. Viktorin ${ }^{1,2,3 *}$, R. Uher ${ }^{4}$, A. Reichenberg ${ }^{1,2}$, S. Z. Levine $^{5}$ and S. Sandin ${ }^{1,2,3}$ \\ ${ }^{1}$ Department of Psychiatry, Icahn School of Medicine, Mount Sinai, New York, NY, USA \\ ${ }^{2}$ The Seaver Autism Center, Research and Treatment at Mount Sinai, New York, NY, USA \\ ${ }^{3}$ Department of Medical Epidemiology and Biostatistics, Karolinska Institutet, Stockholm, Sweden \\ ${ }^{4}$ Department of Psychiatry, Dalhousie University, Halifax, Nova Scotia, Canada \\ ${ }^{5}$ Department of Community Mental Health, University of Haifa, Haifa, Israel
}

Background. Previous studies have examined if maternal antidepressant medication during pregnancy increase the risk of autism spectrum disorder (ASD) in the offspring, but the results have been conflicting.

\begin{abstract}
Methods. In a population-based cohort of 179007 children born in 2006 and 2007 and followed through 2014 when aged 7 and 8, we estimated relative risks (RRs) of ASD and 95\% confidence intervals (CIs) from Cox regression in children exposed to any antidepressant medication during pregnancy, and nine specific antidepressant drugs. Analyses were adjusted for potential confounders and were conducted in the full population sample, and in a clinically relevant subsample of mothers with at least one diagnosis of depression or anxiety during life.
\end{abstract}

Results. The adjusted RR of ASD in children of mothers who used antidepressant medication during pregnancy was estimated at 1.23 (95\% CI 0.96-1.57), and at 1.07 (95\% CI 0.80-1.43) in women with a history of depression or anxiety. Analyses of specific antidepressants initially revealed increased RRs of offspring ASD confined to citalopram and escitalopram (RR: 1.47; 95\% CI 0.92-2.35) and clomipramine (RR: 2.86; 95\% CI 1.04-7.82).

Conclusion. Medication with antidepressants during pregnancy does not appear to be causally associated with an increased risk of ASD in the offspring. Instead, the results suggest that the association is explained by factors related to the underlying susceptibility to psychiatric disorders. Based on these findings, the risk of ASD in the offspring should not be a consideration to withhold treatment with commonly used antidepressant drugs from pregnant women.

Received 10 January 2017; Revised 3 April 2017; Accepted 20 April 2017; First published online 22 May 2017

Key words: Antidepressant, autism, depression, pregnancy, SSRI.

\section{Introduction}

It has been suggested that antidepressant medication in pregnant women may increase the risk of autism spectrum disorder (ASD) in the offspring. This hypothesis has been examined in several studies, yet the results have been mixed; some studies have observed an increased risk (Croen et al. 2011; Rai et al. 2013; Gidaya et al. 2014; Harrington et al. 2014; Boukhris et al. 2016), and others have not (Hviid et al. 2013; Sorensen et al. 2013; Clements et al. 2015; Castro et al. 2016; Malm et al. 2016).

Pharmacotherapy plays a central role in the management of the depressive illness, and untreated depression has been associated with poor health outcomes in both mothers and offspring (Lecrubier, 2001; Marmorstein et al. 2004; O'Hara \& McCabe, 2013;

\footnotetext{
* Address for correspondence: A. Viktorin, Department of Psychiatry, Icahn School of Medicine, Mount Sinai, One Gustave L Levy Place, Box \#1230, New York, NY 10029, USA.

(Email: alexander.viktorin@mssm.edu)
}

Meltzer-Brody \& Stuebe, 2014). With the introduction of selective serotonin re-uptake inhibitors (SSRIs), the last two decades have seen a substantial increase in prescriptions of antidepressant drugs (Olfson \& Marcus, 2009), also in pregnant women (Andrade et al. 2008; Bakker et al. 2008). Although SSRI antidepressants are typically well tolerated (Cipriani et al. 2009), the risk of adverse effects on the developing fetus has not been resolved.

An association between maternal antidepressant medication during pregnancy and offspring ASD could either be due to: (1) a direct effect of the drug or (2) factors that confound the antidepressant treatment and the outcome studied.

Previous reported associations between maternal antidepressant medication during pregnancy and ASD in the offspring have been limited to studies of antidepressant medications as a whole (Croen et al. 2011; Rai et al. 2013; Sorensen et al. 2013; Clements et al. 2015; Castro et al. 2016), or SSRI antidepressants as a whole (Hviid et al. 2013; Gidaya et al. 2014; Harrington et al. 2014; Boukhris et al. 2016; Malm

This is an Open Access article, distributed under the terms of the Creative Commons Attribution licence (http://creativecommons.org/licenses/by/4.0/), which permits unrestricted re-use, distribution, and reproduction in any medium, provided the original work is properly cited. 
et al. 2016). The risk of offspring ASD associated with specific antidepressant drugs is still unknown. If the risk is confined to certain drugs, the proportion of mothers treated with these drugs in prior studies may explain the varying results. Furthermore, recent studies indicate that psychiatric disorders share genetic determinants (International Schizophrenia Consortium et al. 2009; Lichtenstein et al. 2009; Sullivan et al. 2012; Jokiranta et al. 2013). Pregnant women suffering from a mental illness during pregnancy, for which antidepressants may be prescribed, likely carry genetic susceptibility that could be inherited by the child (Jokiranta et al. 2013). As such, the genetic susceptibility, rather than the medication, may completely or partially explain the increased risk of ASD in children of mothers using antidepressants during pregnancy. Yet, previous studies have not thoroughly examined to what extent the risk of offspring ASD may be influenced by underlying susceptibility to mental illness.

The aim of the study was to examine the association between maternal antidepressant medication during pregnancy and ASD in the offspring, investigating any antidepressant medication, and specific antidepressant drugs. We performed an analysis using one of the most comprehensive databases for offspring ASD and parental disorder and medication data available. This allowed us to investigate specific antidepressant drugs used during pregnancy, and use detailed adjustment for parental psychiatric diagnoses. Moreover, we investigated both the full cohort and a clinically relevant sub-sample of mothers with any diagnosis of depression and/or anxiety in their lifetime.

\section{Methods}

\section{Population}

A birth cohort based on all live-born children conceived from July 1, 2005 and born in 2006 and 2007 was established by linkage of Swedish National registers using the unique individual Swedish national registration number (Ludvigsson et al. 2009). Offspring and mothers were identified in the Swedish Medical Birth Register that covers $99 \%$ of all births nation-wide since 1973 and provides information on gestational age at birth that were used to calculate the beginning of pregnancy (Cnattingius et al. 1990). In Sweden, $95 \%$ of all pregnant women receive early second trimester ultrasonography, which provide the gestational age of the fetus with an error margin of \pm 7 days (Hogberg \& Larsson, 1997). The fathers were identified using the Multi-Generation Register (Ekbom, 2011). To be included, the children had to have complete information on gestational age at birth and the identity of the father. The study was approved by the Regional Ethics Committee in Stockholm, Sweden.

\section{Exposures}

The Swedish Prescribed Drug Register holds information on all dispensed prescription drugs in Sweden since July 1,2005 along with drug name, prescription- and dispensation dates, and the Anatomical Therapeutic Chemical Classification System (ATC) code (WHO, 2006; Wettermark et al. 2007). We identified dispensations of all psychotropic drugs prescribed in Sweden, including antidepressants, anxiolytics, stimulants, mood stabilizers, antipsychotics, and sedatives (online Supplementary Tables S1 and S2). The offspring were classified as unexposed to antidepressants if they were born to mothers without any dispensation of an antidepressant with a medication period overlapping the pregnancy. Since the number of antidepressant dispensations was reduced during pregnancy, compared with prior or after (online Supplementary Fig. S1), it was assumed that a single antidepressant dispensation overlapping pregnancy could represent medication that was halted prior the pregnancy. Therefore, antidepressant-exposed offspring were grouped into those born to a mother with either: (1) one single medication dispensation overlapping the pregnancy or (2) two or more dispensations of antidepressants with medication periods overlapping pregnancy (online Supplementary Fig. S2).

\section{Ascertainment of ASD}

The children were followed from birth through 2014 when aged 7 or 8 . A clinically ascertained diagnosis of offspring ASD was identified in the Swedish Patient Register (Ludvigsson et al. 2011; Sandin et al. 2016). This register includes all inpatient psychiatric admissions since 1973 and all outpatient specialist admissions since 2001, and provides admission dates along with the main and eight secondary diagnosis codes in accordance with the International Classification of Disease (ICD). Autism spectrum disorder was defined by having at least one in- or outpatient specialist care admission between birth and end of follow-up at December 31, 2014 with an ICD-10 code according to: F84.0, F84.1, F84.2, F84.3, F84.4, F84.5, F84.8, or F84.9. The ASD diagnoses in the Swedish Patient Register has previously been validated (Idring et al. 2012), and published on extensively (Sandin et al. 2016).

\section{Covariates}

To adjust for potential temporal trends, the birth date of the offspring was included as number of days from January 1, 2005 to the birth date of the child. 
Maternal and paternal age at childbirth was categorized into below 20, 20-29, 30-39, and above 40 years of age. Maternal and paternal susceptibility to mental illness were ascertained based on having at least one psychiatric diagnosis in the Swedish Patient Register within several psychiatric disorder sub-groups at any time in life (online Supplementary Table S3). The father's medication with any psychotropic drugs overlapping the pregnancy was also included, as well as mother's dispensations of other psychotropic medication that overlapped the pregnancy.

\section{Statistical analysis}

Relative risks (RRs) of ASD and the associated Wald-type two-sided 95\% confidence intervals (CIs) were estimated by the hazard ratios from Cox regression models. The Cox regression models were fitted using days since birth as the underlying time scale. Each child was followed from birth until a diagnosis of ASD, death as identified in the Statistics Sweden register of vital statistics, or end of follow-up at December 31, 2014 - whichever came first. The RR of ASD was calculated in: (1) offspring born to mothers with a single-drug dispensation with a medication period overlapping the pregnancy and (2) offspring born to mothers with at least two dispensations with medication periods overlapping the pregnancy, compared with offspring born to mothers without a dispensation with a medication period overlapping the pregnancy.

Analyses were conducted in: (A) the complete sample to provide a public health perspective and in (B) a clinically relevant sub-sample of children born to mothers with at least one diagnosis of depression or anxiety in their lifetime, since most individuals who receive treatment with antidepressants also suffer from those disorders. In this sub-sample, the control group corresponds to mothers who are more likely to be considered for antidepressant treatment, and may share a similar genetic susceptibility of mental illness as the medicated mothers.

First, we examined children of mothers medicated during pregnancy with any type of antidepressant. The RRs of ASD were calculated in a sequence of models with increasing degree of adjustment for potential confounding factors according to: model 1 crude analyses without covariate adjustment; model 2 analyses adjusted for including offspring birthdate, mother's and father's dispenses of other psychotropic medications during pregnancy, and maternal and paternal age; model 3 analyses with additional adjustment for any depressive diagnosis in the mother's life time; and model 4 analyses further adjusted for any diagnosis of specific psychiatric disorder sub-groups in either the mother and/or father's life time. The psychiatric disorder sub-groups included depression, anxiety disorders, schizophrenia, bipolar disorder, substance use disorder, compulsive disorder, attention-deficit hyperactive disorder, ASD, intellectual disability, and any other psychiatric diagnosis (online Supplementary Table S3).

Secondly, we further calculated the RR of ASD among children of mothers treated exclusively with one of the nine most prevalent antidepressants in the sample: sertraline, citalopram and escitalopram, fluoxetine, venlafaxine, paroxetine, clomipramine, amitriptyline and nortriptyline, duloxetine, and mirtazapine, compared with children of mothers not treated with antidepressants during pregnancy. These analyses were adjusted for all included covariates, corresponding to model 4 in the analyses of any antidepressant. For the analyses examining specific drugs, to protect against an inflated error rate as a result of performing many statistical tests, we additionally present multiplicity-corrected $p$ values using the Bonferroni-Holm procedure (Holm, 1979).

All tests of statistically significance were done at the nominal 5\% level of significance. Data management and statistical analyses were done using SAS 9.4 and STATA/IC 14, respectively.

\section{Sensitivity analyses}

The proportional hazards assumption for the Cox regression models was examined using Schoenfeld residuals (Grambsch \& Therneau, 1994). To account for potential within-family correlations in the data due to multiple births from the same parents, we used bootstrap techniques (Efron \& Tibshirani, 1994). The analyses were separately repeated for the outcome autistic disorder (ICD-10 F84.0). Potential sex specificity of associations was tested by analyses of male and female offspring separately. Analyses were also conducted separately for SSRI antidepressants, non-SSRI antidepressants, and non-antidepressant psychotropic drugs. The role of socioeconomic status was examined by including an analysis adjusted for education length as a potential confounder. To further examine the effect of the underlying disorder susceptibility, we compared: (a) children born to mothers treated with antidepressants during pregnancy $(N=3982)$, to $(b)$ children born to mothers with no psychotropic medication but diagnosed with: (1) at least one of type psychiatric disorder, (2) at least two psychiatric disorders, or (3) at least three psychiatric disorders according to online Supplementary Table S3.

\section{Results}

Table 1 presents descriptive data for the cohort. Among the 180444 children conceived from July 1, 2005 and born up until December 31, 2007 in the Medical Birth Register, 1437 (0.7\%) did not have 
Table 1. Cohort subject characteristics

\begin{tabular}{|c|c|c|c|c|c|c|}
\hline \multirow[b]{3}{*}{ Characteristics, no (\%) } & \multicolumn{3}{|c|}{ Full population sample } & \multicolumn{3}{|c|}{ Clinical sub-sample ${ }^{\mathrm{a}}$} \\
\hline & \multicolumn{3}{|c|}{$\begin{array}{l}\text { Antidepressant medication during } \\
\text { pregnancy }\end{array}$} & \multicolumn{3}{|c|}{$\begin{array}{l}\text { Antidepressant medication during } \\
\text { pregnancy }\end{array}$} \\
\hline & Unexposed $^{\mathrm{b}}$ & Exposed $^{\mathrm{c}}$ & Ambiguous $^{\mathrm{d}}$ & Unexposed $^{\mathrm{b}}$ & Exposed ${ }^{c}$ & Ambiguous $^{\mathrm{d}}$ \\
\hline Number of offspring & $172646(96.5)$ & $3982(2.2)$ & $2379(1.3)$ & $14805(79.8)$ & $2617(14.1)$ & $1129(6.1)$ \\
\hline $\begin{array}{l}\text { Offspring diagnosed with autism } \\
\text { spectrum disorder }\end{array}$ & $1524(0.9)$ & $85(2.1)$ & $32(1.4)$ & $282(1.9)$ & $62(2.4)$ & $17(1.5)$ \\
\hline $\begin{array}{l}\text { Offspring diagnosed with autistic } \\
\text { disorder }\end{array}$ & $942(0.6)$ & $41(1.0)$ & $21(0.9)$ & $164(1.1)$ & $29(1.1)$ & $11(1.0)$ \\
\hline $\begin{array}{l}\text { Maternal use of psychotropic medication } \\
\text { other than antidepressants during } \\
\text { pregnancy }\end{array}$ & $1297(0.8)$ & $595(14.9)$ & $143(6.0)$ & $423(2.9)$ & $478(18.3)$ & $97(8.6)$ \\
\hline $\begin{array}{l}\text { Paternal psychotropic medication } \\
\text { during pregnancy }\end{array}$ & $5875(3.4)$ & $437(11.0)$ & $181(7.6)$ & $840(5.7)$ & $314(12.0)$ & $98(8.7)$ \\
\hline \multicolumn{7}{|l|}{ Birth year } \\
\hline 2006 & $73002(42.3)$ & $1588(39.9)$ & $951(40.0)$ & $6381(43.1)$ & $1036(39.6)$ & $443(39.2)$ \\
\hline 2007 & $99644(57.7)$ & $2394(60.1)$ & $1428(60.0)$ & $8424(56.9)$ & $1581(60.4)$ & $686(60.8)$ \\
\hline Maternal psychiatric diagnosis & $23551(13.6)$ & $2868(72.0)$ & $1312(55.2)$ & $14805(100)$ & $2617(100)$ & $1129(100)$ \\
\hline Paternal psychiatric diagnosis & $18201(10.5)$ & $836(21.0)$ & $436(18.3)$ & $3056(20.6)$ & $623(23.8)$ & $258(22.9)$ \\
\hline \multicolumn{7}{|l|}{ Maternal age (years) at delivery } \\
\hline$<20$ & $3367(2.0)$ & $61(1.5)$ & $59(2.5)$ & $706(4.8)$ & $48(1.8)$ & $47(4.2)$ \\
\hline $20-29$ & $73009(42.3)$ & $1585(39.8)$ & $975(41.0)$ & 7239 (48.9) & $1119(42.8)$ & $505(44.7)$ \\
\hline $30-39$ & $91716(52.5)$ & $2123(53.3)$ & $1235(51.9)$ & $6374(43.1)$ & $1316(50.3)$ & $532(47.1)$ \\
\hline $40+$ & $5554(3.2)$ & $213(5.35)$ & $110(4.6)$ & $486(3.3)$ & $134(5.1)$ & $45(4.0)$ \\
\hline \multicolumn{7}{|l|}{ Paternal age (years) at delivery } \\
\hline$<20$ & $984(0.6)$ & $21(0.5)$ & $17(0.7)$ & $246(1.7)$ & $15(0.6)$ & $12(1.1)$ \\
\hline $20-29$ & $47994(27.8)$ & $1163(29.2)$ & $743(31.2)$ & $5337(36.1)$ & $840(32.1)$ & $406(36.0)$ \\
\hline $30-39$ & $100624(58.3)$ & $2160(54.2)$ & $1259(52.9)$ & $7240(48.9)$ & $1337(51.1)$ & $568(50.3)$ \\
\hline $40+$ & $23044(13.4)$ & $638(16.0)$ & $360(15.1)$ & $1982(13.4)$ & 425 (16.2) & $143(12.7)$ \\
\hline \multicolumn{7}{|l|}{ Offspring sex } \\
\hline Males & 88858 (51.5) & $2043(51.3)$ & $1232(51.8)$ & 7517 (50.8) & 1349 (51.5) & $585(51.8)$ \\
\hline Females & 83788 (48.5) & 1939 (48.7) & $1147(48.2)$ & 7288 (49.2) & $1268(48.5)$ & $544(48.2)$ \\
\hline
\end{tabular}

${ }^{a}$ A clinically relevant sub-sample consisting of 18551 children, of which 361 had been diagnosed with autism spectrum disorder. All mothers, both medicated and non-medicated, had at least one diagnosis of depression or an anxiety disorder in their lifetime (online Supplementary Table S3). Thereby, the offspring of mothers with medication during pregnancy is contrasted with offspring of mothers that may share similar underlying factors.

${ }^{\mathrm{b}}$ Children of mothers with no antidepressant dispensation with a medication period overlapping pregnancy.

${ }^{c}$ Children of mothers with at least two antidepressant dispensations with medication periods overlapping pregnancy (online Supplementary Fig. S1 examples A1 and A2).

${ }^{\mathrm{d}}$ Children of mothers with a single antidepressant dispensation with a medication period overlapping pregnancy.

complete data and were excluded from the statistical analyses. Table 1 presents descriptive statistics for the 179007 included children and their parents. At least one diagnosis of any psychiatric illness was observed in $47204(13.2 \%)$ parents. Autism spectrum disorder was observed in $1641(0.9 \%)$ of the children, and among those, $1004(61.0 \%)$ had a diagnosis of autistic disorder. Among the offspring, 2379 (1.3\%) were born to a mother with a single antidepressant dispensation with a medication period overlapping pregnancy, and $3982(2.2 \%)$ were born to a mother with at least two antidepressant dispensations with medication periods overlapping pregnancy.

Due to the ambiguity of drug exposure in children to mothers with only a single antidepressant dispensation overlapping pregnancy, the results focus on the children with at least two dispensations overlapping pregnancy, as compared with children without exposure to antidepressants. Results in the offspring of mothers with only a single dispensation overlapping pregnancy are presented in the online supplement (online Supplementary Fig. S3). 
Table 2. Relative risks of autism spectrum disorder due to exposure to any type of antidepressants ${ }^{\mathrm{a}}$

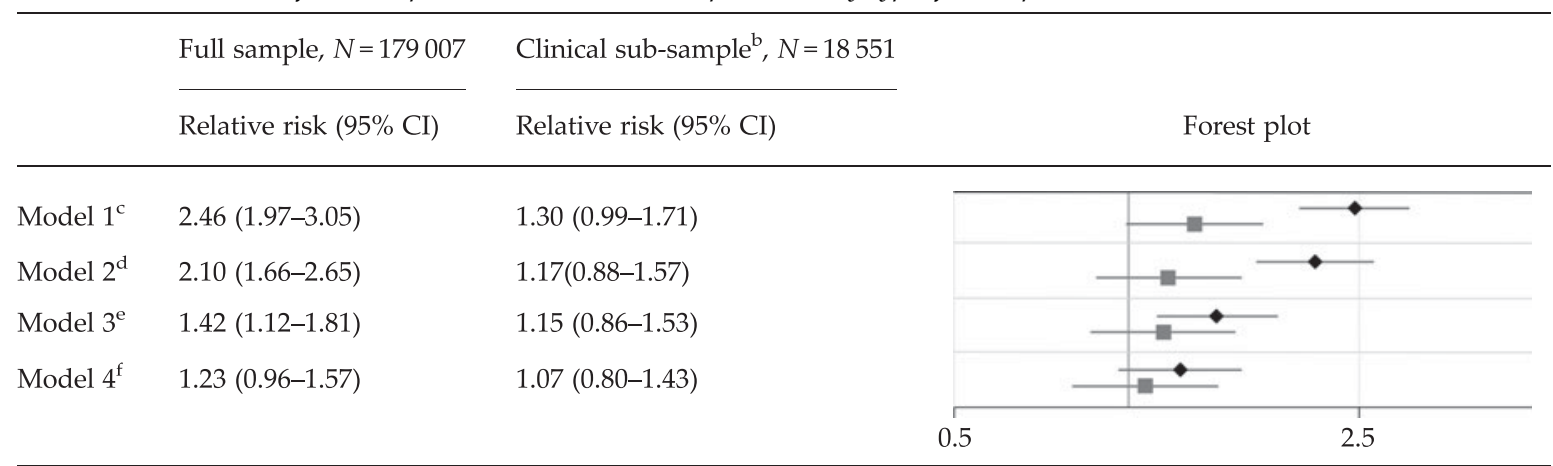

ASD, autism spectrum disorder; CI, confidence interval.

a The sample consists of 179,007 children born during 2006 and 2007, of which 1,641 had been diagnosed with ASD. The figure presents relative risks of ASD and two-sided 95\% confidence intervals in children of mothers with at least two dispensations of a specific antidepressant drug overlapping the pregnancy, compared with unexposed children. The analyses are adjusted for all included covariates, as in model 4 in Figure 1.

${ }^{\mathrm{b}}$ The clinical sub-sample consists of 18551 children, of which 361 had been diagnosed with autism spectrum disorder. All mothers, both medicated and non-medicated, had at least one diagnosis of depression or an anxiety disorder in their lifetime (online Supplementary Table S3). Thereby, the offspring of mothers with medication during pregnancy is contrasted with offspring of mothers that may share similar underlying factors.

${ }^{\mathrm{c}}$ Analyses not adjusted for covariates.

${ }^{\mathrm{d}}$ Analyses adjusted for birthdate, maternal and paternal age, the father's psychotropic medication that overlapped the pregnancy, and the mother's one-time dispensations of psychotropic medication that overlapped the pregnancy.

e Analyses adjusted for the factors listed in footnote ${ }^{\mathrm{d}}$, and for any diagnosis of depression in the mother's lifetime (yes/ no) (see online Supplementary Table S3 for specific diagnosis codes).

${ }^{\mathrm{f}}$ Analyses adjusted for the factors listed in footnote ${ }^{\mathrm{d}}$, and for any diagnosis of specific psychiatric disorder sub-groups in either the mother and/or father's life time (yes/no), including depression, anxiety disorders, substance use disorder, bipolar disorder, compulsive disorder, attention deficit hyperactivity disorder, autism spectrum disorder, intellectual disability, schizophrenia, and 'other psychiatric diagnosis' (see Supplementary Table S3 for specific diagnosis codes).

\section{Risk of ASD}

In the full population sample, the crude RR of ASD in children of mothers with at least two dispensations of antidepressants overlapping pregnancy compared with unexposed children was estimated at $2.46(95 \%$ CI 1.97-3.05). With adjustment for all included potential confounders, the RR was reduced to 1.23 (95\% CI 0.96-1.57). In analyses confined to children of mothers with at least one diagnosis of depression or anxiety in their lifetime, the crude RR of ASD in children of mothers with at least two dispensations of antidepressants overlapping pregnancy was estimated at 1.30 (95\% CI 0.99-1.71). With adjustment for potential confounders, the RR was reduced to $1.07(0.80-1.43)$ (Table 2).

\section{Risk of ASD associated with specific medications}

In the full population sample, the adjusted RRs of ASD in children of mothers with at least two dispensations of specific antidepressants overlapping pregnancy compared with unexposed children was estimated at 1.35 (95\% CI 0.87-2.08) for sertraline, $1.71 \quad(95 \%$ CI $1.16-2.51)$ for citalopram and escitalopram, 1.04 (95\% CI 0.53-2.02) for fluoxetine, 1.22 (95\% CI 0.54-2.75) for venlafaxine, 1.40 (95\% CI 0.52-3.76) for paroxetine, 3.27 (95\% CI 1.33-8.00) for clomipramine, 0.59 (95\% CI 0.08-4.20) for amitriptyline and nortriptyline, and 1.53 (95\% CI 0.38-6.23) for mirtazapine (Table 3).

Within the sub-sample where both the medicated and non-medicated women had at least one diagnosis of depression or anxiety in their lifetime, the adjusted RRs of ASD in children of mothers with at least two dispensations of specific antidepressants overlapping pregnancy compared with unexposed children was estimated at 1.17 (95\% CI 0.71-1.95) for sertraline, 1.47 (95\% CI 0.92-2.35) for citalopram and escitalopram, 1.08 (95\% CI 0.53-2.21) for fluoxetine, 0.88 (95\% CI 0.32-2.38) for venlafaxine, 1.21 (95\% CI 0.38-3.80) for paroxetine, 2.86 (95\% CI 1.04-7.82) for clomipramine, and 1.00 (95\% CI 0.14-7.24) for mirtazapine (Table 3).

None of the analyses examining specific drugs, in the full sample and in the clinically relevant subsample, revealed statistically significant $p$ values after multiplicity correction using the Bonferroni-Holm procedure (Holm, 1979). 
Table 3. Relative risks of autism spectrum disorder due to exposure to specific antidepressants ${ }^{\mathrm{a}}$

\begin{tabular}{|c|c|c|c|c|c|c|c|c|c|c|c|c|}
\hline \multirow[b]{2}{*}{ Medication } & \multicolumn{5}{|c|}{ Full sample, $N=179007$} & \multicolumn{5}{|c|}{ Clinical sub-sample ${ }^{\mathrm{b}}, N=18551$} & \multirow{2}{*}{\multicolumn{2}{|c|}{ Forest plot }} \\
\hline & $\begin{array}{l}\text { Exposed } \\
\text { children }\end{array}$ & $\begin{array}{l}\text { Exposed } \\
\text { children } \\
\text { with } \\
\text { ASD }(\%)\end{array}$ & $\begin{array}{l}\text { Relative risk } \\
(95 \% \mathrm{CI})\end{array}$ & $p$ value & $\begin{array}{l}\text { Bonferroni- } \\
\text { Holm } \\
\text { corrected } \\
p \text { value }^{\mathrm{c}}\end{array}$ & $\begin{array}{l}\text { Exposed } \\
\text { children }\end{array}$ & $\begin{array}{l}\text { Exposed } \\
\text { children } \\
\text { with } \\
\text { ASD (\%) }\end{array}$ & $\begin{array}{l}\text { Relative risk } \\
(95 \% \mathrm{CI})\end{array}$ & $p$ value & $\begin{array}{l}\text { Bonferroni- } \\
\text { Holm } \\
\text { corrected } \\
p \text { value }^{\mathrm{c}}\end{array}$ & & \\
\hline Sertraline & 1016 & $22(2.2)$ & $1.35(0.87-2.08)$ & 0.186 & 1.000 & 672 & $16(2.4)$ & $1.17(0.71-1.95)$ & 0.485 & 1.000 & & $\bullet$ \\
\hline $\begin{array}{l}\text { Citalopram } \\
\text { and } \\
\text { escitalopram }\end{array}$ & 1097 & $28(2.6)$ & $1.71(1.16-2.51)$ & 0.006 & 0.090 & 639 & $19(3.0)$ & $1.47(0.92-2.35)$ & 0.100 & 1.000 & & $\because$ \\
\hline Fluoxetine & 458 & $9(2.0)$ & $1.04(0.53-2.02)$ & 0.919 & 1.000 & 327 & $8(2.4)$ & $1.08(0.53-2.21)$ & 0.783 & 1.000 & & 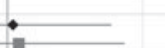 \\
\hline Venlafaxine & 266 & $6(2.3)$ & $1.22(0.54-2.75)$ & 0.632 & 1.000 & 195 & $4(2.1)$ & $0.88(0.32-2.38)$ & 0.819 & 1.000 & 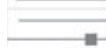 & $\rightarrow$ \\
\hline Paroxetine & 173 & $4(2.3)$ & $1.40(0.52-3.76)$ & 0.516 & 1.000 & 108 & $3(2.8)$ & $1.21(0.38-3.80)$ & 0.714 & 1.000 & & $=$ \\
\hline Clomipramine & 91 & $5(5.5)$ & $3.27(1.33-8.00)$ & 0.010 & 0.140 & 68 & $4(5.9)$ & $2.86(1.04-7.82)$ & 0.034 & 0.442 & & 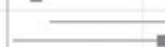 \\
\hline $\begin{array}{l}\text { Amitriptyline } \\
\text { and } \\
\text { nortriptyline }\end{array}$ & 133 & $1(0.8)$ & $0.59(0.08-4.20)$ & 0.597 & 1.000 & 40 & $0(0)$ & $N A$ & NA & $N A$ & $\rightarrow$ & \\
\hline Duloxetine & 52 & $0(0)$ & $N A$ & $N A$ & $\mathrm{Na}$ & 37 & $0(0)$ & $N A$ & $N A$ & $N A$ & & \\
\hline \multirow[t]{2}{*}{ Mirtazapine } & 62 & $2(3.2)$ & $1.53(0.38-6.23)$ & 0.376 & 1.000 & 36 & $1(2.8)$ & $1.00(0.14-7.24)$ & 1.000 & 1.000 & $\bar{z}$ & - \\
\hline & & & & & & & & & & & .5 & 2.5 \\
\hline
\end{tabular}

ASD, autism spectrum disorder; N, number of children; CI, confidence interval; NA, not available.

${ }^{a}$ The sample consists of 179007 children born during 2006 and 2007, of which 1641 had been diagnosed with ASD. The figure presents relative risks of ASD and two-sided 95\% CIs in children of mothers with at least two dispensations of a specific antidepressant drug overlapping the pregnancy, compared with unexposed children. The analyses are adjusted for all included covariates, as in model 4 in Table 2.

${ }^{b}$ The clinical sub-sample consists of 18551 children, of which 361 had been diagnosed with ASD. All mothers, both medicated and non-medicated, had at least one diagnosis of depression or an anxiety disorder in their lifetime (online Supplementary Table S3). Thereby, the offspring of mothers with medication during pregnancy is contrasted with offspring of mothers that may share similar underlying factors.

${ }^{\mathrm{c}}$ Multiplicity-corrected $p$ values using the Bonferroni-Holm procedure: Holm (1979). A simple sequentially rejective multiple test procedure. Scandinavian Journal of Statistics 6, pp. 65-70. 


\section{Sensitivity analyses}

Inspection of the Schonfeld residuals did not suggest any violation of the proportional hazards assumption (online Supplementary Fig. S4). Analyses confined to specifically SSRI antidepressants, non-SSRI antidepressants, and non-antidepressant psychotropic drugs revealed results quantitatively similar to the analyses of any antidepressant (online Supplementary Fig. S5). Confidence intervals of selected point estimates (RRs) calculated using bootstrap revealed close to identical results as the parametric Wald estimates (online Supplementary Fig. S6). Analyses confined to autistic disorder showed results similar to that of ASD (online Supplementary Fig. S7). Analyses of male and female offspring separately did not reveal any sex-specific association (online Supplementary Fig. S8). The complementary analysis adjusting for education as a marker of socioeconomic status did not affect our results (online Supplementary Fig. S9). Analyses with comparison groups without psychotropic medication, but with increasing number of diagnosed psychiatric diagnoses revealed that the RR of ASD in the offspring was closely correlated with the number of different psychiatric disorders diagnosed in the mothers (online Supplementary Figs. S11 and S12).

\section{Discussion}

In this population-based, prospective cohort study of 179007 children and their parents, we observed an increased RR of ASD in offspring of mothers treated with antidepressant medication during pregnancy compared with offspring of mothers not treated with antidepressants during pregnancy. However, detailed adjustments for confounding by parental psychiatric liability attenuated this risk. Moreover, when the analyses were restricted to mothers ever diagnosed with depression or anxiety, a likely target group for antidepressant medication, the association between antidepressants in pregnancy and ASD were attenuated even further and close to none.

To our knowledge, this is the first study to examine the RR of offspring ASD associated with specific types of antidepressant drugs compared with children unexposed to antidepressants. Among the nine studied drugs, only the SSRI antidepressants citalopram and escitalopram, and the tricyclic antidepressant clomipramine displayed an increased RR of ASD in the offspring. While these findings could suggest that these particular medications may have a causal effect associated with increased risk of ASD in the offspring, these positive associations could also be due to residual confounding. Citalopram and escitalopram have similar mechanisms of action as sertraline and paroxetine, yet only citalopram and citalopram displayed an elevated RR of ASD in the offspring. Furthermore, this increase in RR was modest, not statistically significant in the clinically relevant subsample, and not statistically different from the results of the other specific antidepressants studied. The results of the present study strongly suggest that the associations between antidepressant treatment during pregnancy and offspring ASD are gradually attenuated with increasing covariate adjustment. Although the present study adjusts for a broad set of factors that may confound the association, the ability to capture confounding is not complete. Mothers treated with different antidepressant medications may be different in other aspects that the current study cannot adjust for. This is exemplified by clomipramine that is not recommended as a first line of treatment of depression, which therefor may be given to women with a more severe or complex form of depression. Further analysis of this treatment group indeed revealed an increased prevalence of both compulsive disorder and schizophrenia compared with women treated with other antidepressants (online Supplementary Fig. S10).

To further investigate the role of psychiatric disorders in the association between antidepressant treatment during pregnancy and ASD in the offspring, we compared children exposed to antidepressants to children of mothers not using psychotropic medication during pregnancy, but with increasing mean number of psychiatric disorders (online Supplementary Fig. S11). These analyses revealed that a higher mean number of different psychiatric disorders diagnosed during the mothers' lifetime was correlated with the RR of ASD in the offspring. This was also observed in analyses of specific antidepressant drugs (online Supplementary Fig. S12)

Nevertheless, the relationship between treatment with specific antidepressants during pregnancy and the risk of ASD in the offspring should be examined in additional samples to shed more light on the association and the specific underlying disorders.

Prior research into the association between maternal antidepressant medication during pregnancy and offspring ASD has delivered mixed and inconclusive results (Croen et al. 2011; Hviid et al. 2013; Rai et al. 2013; Sorensen et al. 2013; Gidaya et al. 2014; Harrington et al. 2014; Clements et al. 2015; Boukhris et al. 2016; Castro et al. 2016; Malm et al. 2016). The present findings may resolve this earlier ambiguity. Our findings of attenuated associations with incremental covariate adjustment are in line with several earlier studies (Hviid et al. 2013; Sorensen et al. 2013; Clements et al. 2015; Castro et al. 2016; Malm et al. 2016). The present findings are, however, at odds with number of previous studies that report statistically significant associations. A study of 1054 Canadian children 
with ASD (Boukhris et al. 2016) reported an unadjusted RR of ASD following SSRI treatment ( $R R=2.27 ; 95 \% \mathrm{CI}$ 1.48-3.46) that were partially attenuated after adjustments for factors, including maternal history of depression ( $R R=1.75 ; 95 \%$ CI 1.03-2.97). Similarly sized RRs have also been reported in a Californian sample of 298 children with ASD (RR=2.2; 95\% CI 1.2-4.3) (Croen et al. 2011), in a Danish sample of 5215 children with ASD (adjusted RR $=1.8$; 95\% CI 1.4-2.3) (Gidaya et al. 2014), in a US study of 421 male cases with ASD (adjusted $\mathrm{RR}=2.91$; 95\% CI 1.07-7.93) (Harrington et al. 2014), and in a Swedish study of 4429 cases with ASD (RR=3.34; 95\% CI 1.50-7.47) (Rai et al. 2013).

The discrepancies between the results of previous studies and the present study could be due to several factors. If the present findings of different psychiatric disorder profiles in mothers treated with specific antidepressants (online Supplementary Figs. S11 and S12) was comparable in previous studies, the proportion of these specific antidepressant drugs could explain the mixed results in previous studies. However, the limited adjustment for the parents' mental illnesses in previous studies may also explain the discrepancies.

Strengths of the study include a large prospective population-based sample of children and their parents with close to complete healthcare data coverage. Any estimate of prevalence of ASD is highly dependent on the birth year, follow-up time, and sex distribution in the sample, still our estimates agree well with other studies in the Nordic countries and in the USA (Idring et al. 2012; Sandin et al. 2016). Data from a healthcare system with equal access limit the risk of selection biases. Inclusion of children born during a limited period reduces potential confounding by factors that may vary over time. Examination of any diagnosis of mental illness in the parents' lifetime allowed detailed adjustment for confounding due to, e.g., genetic liability. The requirement of at least two dispensations overlapping the pregnancy further increased the specificity of the exposure. Furthermore, supplementary analyses of antidepressants divided into specifically SSRI antidepressants and non-SSRI antidepressants, and nonantidepressant psychotropic drugs all revealed similar findings, lending further support to the interpretation that the mental illness confounds the association between maternal antidepressant medication during pregnancy and increased risk of ASD in offspring.

However, the findings should also be interpreted in light of some limitations. Overall, our study summarizes the results of a multitude of statistical tests, which always increase the risk of erroneous rejection of a specific hypothesis. When applying the Bonferroni-Holm procedure in the comparison of specific drugs compared with 'no treatment of antidepressants', no drugs were at a statistically significantly higher risk. Although drugs recorded in the Prescribed Drug Register have been prescribed and collected, we cannot be entirely sure to what extent the medication was actually consumed. To address this limitation, we divided the exposed children into those born to a mother with only a single dispensation, and those with at least two dispensations overlapping the pregnancy. The study focused on those with at least two dispensations, as a continuous dispensation pattern was assumed to better reflect an ongoing treatment. Although we adjusted for both parents' education level at childbirth, we could not adjust for the income of the family. While education level had no effect on the association in our Swedish sample, this may not be the case in cohorts from other countries. Moreover, the Patient Register does not provide information from the primary care, and as such, any diagnoses confined to a primary care setting will go undetected. Still, this will not affect the study's ability to detect children with an ASD, as they are referred to a specialist that is covered by the Patient Register. The lack of primary care data may, however, affect the study's ability to capture psychiatric diagnoses as measure of mental illness among the parents, and consequently the ability to adjust for potential confounding due to underlying mental illness diagnosed in a primary care setting only. Antidepressant prescriptions are accompanied with a diagnosis in Sweden, yet supplemental analyses show that about $25 \%$ of mothers treated with an antidepressant during pregnancy lack a psychiatric diagnosis in the Patient Register (online Supplementary Fig. S13), which could indicate residual confounding. Moreover, the severity of the psychiatric disorders investigated was not known, nor if the mothers experienced ongoing episodes of the lifetime diagnosed disorders. It is likely that mothers treated with antidepressants during pregnancy experienced a more severe disorder than unmedicated mothers with a lifetime diagnosis of depression or anxiety. Finally, the current study cohort was restricted to children born in 2006 and 2007, and therefore is underpowered to perform family-control analyses, which can be valuable to address potential residual confounding due to shared familial and genetic factors.

\section{Conclusion}

Medication with antidepressants during pregnancy does not appear to be causally associated with an increased risk of ASD in the offspring. Instead, the results suggest that the association is explained by factors related to the underlying susceptibility to psychiatric disorders. Based on these findings, the risk of ASD in the offspring should not be a consideration to 
withhold treatment with commonly used antidepressant from pregnant women.

\section{Supplementary material}

The supplementary material for this article can be found at https://doi.org/10.1017/S0033291717001301.

\section{Acknowledgment}

This study was supported by grants from the National Institutes of Health; grant HD073978 from the Eunice Kennedy Shriver National Institute of Child Health and Human Development, National Institute of Environmental Health Sciences, and National Institute of Neurological Disorders and Stroke; grant MH097849 from the National Institute of Mental Health; by the Beatrice and Samuel A. Seaver Foundation; by the Fredrik and Ingrid Thuring Foundation; and by the Swedish Society of Medicine. The sponsors of the study had no role in study design, data collection, data analysis, data interpretation, writing of the report, or in the decision to submit the paper for publication. The corresponding author had full access to all data in the study and had final responsibility for the decision to submit for publication.

\section{Declaration of Interest}

None.

\section{References}

Andrade SE, Raebel MA, Brown J, Lane K, Livingston J, Boudreau D, Rolnick SJ, Roblin D, Smith DH, Willy ME, Staffa JA, Platt R (2008). Use of antidepressant medications during pregnancy: a multisite study. American Journal of Obstetrics \& Gynecology 198, 194e1-194e5.

Bakker MK, Kolling P, van den Berg PB, de Walle HE, de Jong van den Berg LT (2008). Increase in use of selective serotonin reuptake inhibitors in pregnancy during the last decade, a population-based cohort study from the Netherlands. British Journal of Clinical Pharmacology 65, 600-606.

Boukhris T, Sheehy O, Mottron L, Bérard A (2016). Antidepressant use during pregnancy and the risk of autism spectrum disorder in children. JAMA Pediatrics 170, 117-124.

Castro V, Kong S, Clements C, Brady R, Kaimal A, Doyle A, Robinson E, Churchill S, Kohane I, Perlis R (2016). Absence of evidence for increase in risk for autism or attention-deficit hyperactivity disorder following antidepressant exposure during pregnancy: a replication study. Translational Psychiatry 6, e708.

Cipriani A, Furukawa TA, Salanti G, Geddes JR, Higgins JP, Churchill R, Watanabe N, Nakagawa A, Omori IM, McGuire H, Tansella M, Barbui C (2009). Comparative efficacy and acceptability of 12 new-generation antidepressants: a multiple-treatments meta-analysis. Lancet 373, 746-758.

Clements CC, Castro VM, Blumenthal SR, Rosenfield HR, Murphy SN, Fava M, Erb JL, Churchill SE, Kaimal AJ, Doyle AE (2015). Prenatal antidepressant exposure is associated with risk for attention-deficit hyperactivity disorder but not autism spectrum disorder in a large health system. Molecular Psychiatry 20, 727-734.

Cnattingius S, Ericson A, Gunnarskog J, Kallen B (1990). A quality study of a medical birth registry. Scandinavian Journal of Social Medicine 18, 143-148.

Croen LA, Grether JK, Yoshida CK, Odouli R, Hendrick V (2011). Antidepressant use during pregnancy and childhood autism spectrum disorders. Archives of General Psychiatry 68, 1104-1112.

Efron B, Tibshirani RJ (1994). An Introduction to the Bootstrap. Chapman and Hall/CRC Press: London.

Ekbom A (2011). The Swedish multi-generation register. Methods in Molecular Biology 675, 215-220.

Gidaya NB, Lee BK, Burstyn I, Yudell M, Mortensen EL, Newschaffer CJ (2014). In utero exposure to selective serotonin reuptake inhibitors and risk for autism spectrum disorder. Journal of Autism and Developmental Disorders 44, 2558-2567.

Grambsch PM, Therneau TM (1994). Proportional hazards tests and diagnostics based on weighted residuals. Biometrika 81, 515-526.

Harrington RA, Lee L-C, Crum RM, Zimmerman AW, Hertz-Picciotto I (2014). Prenatal SSRI use and offspring with autism spectrum disorder or developmental delay. Pediatrics 133, e1241-e1248.

Hogberg U, Larsson N (1997). Early dating by ultrasound and perinatal outcome. A cohort study. Acta Obstetricia et Gynecologica Scandinavica 76, 907-912.

Holm S (1979). A simple sequentially rejective multiple test procedure. Scandinavian Journal of Statistics 6, 65-70.

Hviid A, Melbye M, Pasternak B (2013). Use of selective serotonin reuptake inhibitors during pregnancy and risk of autism. New England Journal of Medicine 369, 2406-2415.

Idring S, Rai D, Dal H, Dalman C, Sturm H, Zander E, Lee BK, Serlachius E, Magnusson C (2012). Autism spectrum disorders in the Stockholm Youth Cohort: design, prevalence and validity. PLoS ONE 7, e41280.

International Schizophrenia Consortium, Purcell SM, Wray NR, Stone JL, Visscher PM, O'Donovan MC, Sullivan PF, Sklar P (2009). Common polygenic variation contributes to risk of schizophrenia and bipolar disorder. Nature 460, 748-752.

Jokiranta E, Brown AS, Heinimaa M, Cheslack-Postava K, Suominen A, Sourander A (2013). Parental psychiatric disorders and autism spectrum disorders. Psychiatry Research 207, 203-211.

Lecrubier Y (2001). The burden of depression and anxiety in general medicine. Journal of Clinical Psychiatry 62(Suppl. 8), 4-9; discussion 10-1.

Lichtenstein P, Yip BH, Bjork C, Pawitan Y, Cannon TD, Sullivan PF, Hultman CM (2009). Common genetic determinants of schizophrenia and bipolar disorder in Swedish families: a population-based study. Lancet 373, 234-239. 
Ludvigsson JF, Andersson E, Ekbom A, Feychting M, Kim JL, Reuterwall C, Heurgren M, Olausson PO (2011). External review and validation of the Swedish national inpatient register. BMC Public Health 11, 450.

Ludvigsson JF, Otterblad-Olausson P, Pettersson BU, Ekbom A (2009). The Swedish personal identity number: possibilities and pitfalls in healthcare and medical research. European Journal of Epidemiology 24, 659-667.

Malm H, Brown AS, Gissler M, Gyllenberg D, Hinkka-Yli-Salomaki S, McKeague IW, Weissman M, Wickramaratne P, Artama M, Gingrich JA, Sourander A (2016). Gestational exposure to selective serotonin reuptake inhibitors and offspring psychiatric disorders: a national register-based study. Journal of the American Academy of Child and Adolescent Psychiatry 55, 359-366.

Marmorstein NR, Malone SM, Iacono WG (2004).

Psychiatric disorders among offspring of depressed mothers: associations with paternal psychopathology. American Journal of Psychiatry 161, 1588-1594.

Meltzer-Brody S, Stuebe A (2014). The long-term psychiatric and medical prognosis of perinatal mental illness. Best Practice \& Research Clinical Obstetrics \& Gynaecology 28, 49-60.

O'Hara MW, McCabe JE (2013). Postpartum depression: current status and future directions. Annual Review of Clinical Psychology 9, 379-407.

Olfson M, Marcus SC (2009). National patterns in antidepressant medication treatment. Archives of General Psychiatry 66, 848-856.
Rai D, Lee BK, Dalman C, Golding J, Lewis G, Magnusson C (2013). Parental depression, maternal antidepressant use during pregnancy, and risk of autism spectrum disorders: population based case-control study. BMJ 346, f2059.

Sandin S, Schendel D, Magnusson P, Hultman C, Suren P, Susser E, Gronborg T, Gissler M, Gunnes N, Gross R, Henning $M$, Bresnahan $M$, Sourander A, Hornig $M$, Carter K, Francis R, Parner E, Leonard H, Rosanoff M, Stoltenberg C, Reichenberg A (2016). Autism risk associated with parental age and with increasing difference in age between the parents. Molecular Psychiatry 21, 693-700.

Sorensen MJ, Gronborg TK, Christensen J, Parner ET, Vestergaard M, Schendel D, Pedersen LH (2013). Antidepressant exposure in pregnancy and risk of autism spectrum disorders. Clinical Epidemiology 5, 449-459.

Sullivan PF, Daly MJ, O'Donovan M (2012). Genetic architectures of psychiatric disorders: the emerging picture and its implications. Nature Reviews Genetics 13, 537-551.

Wettermark B, Hammar N, Fored CM, Leimanis A, Otterblad Olausson P, Bergman U, Persson I, Sundstrom A, Westerholm B, Rosen M (2007). The new Swedish Prescribed Drug Register - opportunities for pharmacoepidemiological research and experience from the first six months. Pharmacoepidemiology and Drug Safety 16, 726-735.

WHO (2006). The Anatomical Therapeutic Chemical Classification System with Defined Daily Doses (ATC/DDD). WHO: Oslo. 